\title{
THREE WEEKS OF COMBINED RESISTED AND ASSISTED IN-WATER TRAINING FOR ADOLESCENT SPRINT BACKSTROKE SWIMMING: A CASE STUDY
}

original paper

() Wroclaw University of Health and Sport Sciences

DOI: https://doi.org/10.5114/hm.2022.110123

\author{
KHALID ABDULLAH SALEH AL-SHDOUKHI ${ }^{1}$, CARL PETERSEN ${ }^{2 \oplus}$, \\ JENNY CLARKE ${ }^{2 \oplus}$ \\ ${ }^{1}$ Department of Sports Science and Physical Activity, University of Hail, Hail, Saudi Arabia \\ ${ }^{2}$ School of Health Sciences, University of Canterbury, Christchurch, New Zealand
}

\begin{abstract}
Purpose. Resisted and assisted in-water training methods are often employed in swimming training but their effectiveness remains unsubstantiated for different strokes and age groups. The study aim was to quantify the effects of a 3-week combined assisted and resisted in-water training program on 50- and 100-m adolescent backstroke performance.

Methods. In addition to regular swimming training, 9 (5 male, 4 female; age: $15.4 \pm 1.7$ years; 50-m backstroke FINA points: $346 \pm 142$ ) competitive backstroke swimmers performed a combined in-water machine-resisted and bungee-assisted training program 3 days/week with 3 resisted and 3 assisted 25-m sprints per session. Before and after the 3-week training, 50- and 100-m backstroke time trials were undertaken, with stroke rate, heart rate, and rating of perceived exertion collected. Results. There was a significant small improvement in 100 -m backstroke times $(3.4 \pm 3.4 \%$ faster; $E S=0.27, p<0.01)$, but only a trivial improvement in 50 -m backstroke times $(1.0 \pm 3.1 \%$ faster; $E S=0.07, p=0.19)$. Females had substantially greater improvements than males in both $50-\mathrm{m}$ ( $2.4 \pm 2.7 \%$ faster vs. $-0.2 \pm 3.2 \%$ slower $)$ and 100 -m $(5.1 \pm 2.6 \%$ faster vs. $2.0 \pm 3.5 \%$ faster) backstroke time trials, but with the small sample size, this warrants further investigation.

Conclusions. We demonstrated that adding a 3-week combined in-water resisted and assisted training was likely more beneficial for the longer 100-m distance; females seemed to benefit more than males.
\end{abstract}

Key words: strength, physiology, youth sport

\section{Introduction}

To improve maximum sprint swimming performance, coaches employ a variety of training apparatus and interventions to target physiological and technical parameters. Two of the most common methods are in-water resistance and assistance training. Assisted sprinting, also known as overspeed training, can be performed by having a swimmer wear a belt attached to an extended bungee cord and then having a coach pull the swimmer in towards them while the swimmer simultaneously swims as fast as possible [1]. A novel way of implementing resisted sprint swimming is for a swimmer wearing a belt to be attached to a variable resistance machine positioned at the end of the lane. This type of machine has the benefit of requiring a swimmer to overcome an individualized yet set con- sistent resistance throughout the entire swimming sprint.

To date, backstroke resisted and assisted in-water training has received negligible research attention. However, combining swimming with in-water resisted or assisted sprint training for freestyle swimming has been reported as being more efficient than traditional freestyle swimming training alone [2, 3]. These authors noted that, after in-water assisted sprinting, freestyle swimmers increased their stroke rate (SR) $(p<0.05)$ and reduced their stroke length (SL) $(p<0.05)$; however, these changes resulted in an unchanged swimming velocity. Comparatively, resisted sprint training has been found to have had a larger positive effect on muscle strength, swimming performance, and stroke technique than assisted sprint training $[2,4,5]$. Nevertheless, other studies have not found any benefit

Correspondence address: Carl Petersen, School of Health Sciences, University of Canterbury, Private Bag 4800, Christchurch 8140, New Zealand, e-mail: carl.petersen@canterbury.ac.nz, https://orcid.org/0000-0003-3872-914X

Received: December 15, 2020

Accepted for publication: August 11, 2021

Citation: Al-Shdoukhi KAS, Petersen C, Clarke J. Three weeks of combined resisted and assisted in-water training for adolescent sprint backstroke swimming: a case study. Hum Mov. 2022;23(4):133-139; doi: https://doi.org/10.5114/hm.2022.110123. 
with resisted swim training for 50-m [6] or 100-, 200-, or 400-m freestyle performance [7, 8]. Still, the freestyle stroke is faster than backstroke as a result of a higher stroke frequency, due to a shorter propulsive and recovery phase duration [9]. Differences in SR may result in differences in the effectiveness of resisted and assisted training interventions across types of stroke. The effectiveness of resisted or assisted training in young swimmers remains undocumented, despite research studies suggesting that pre-adolescents have similar training responses to elite athletes, albeit with adaptions of different magnitudes [10-13]. In summary, we believe that the effectiveness of combining resisted and assisted in-water sprint training in adolescent backstroke performance has not been adequately examined. To address this, we aimed to assess the effectiveness of combined resisted and assisted training in 50- and 100-m adolescent backstroke performance.

\section{Material and methods}

\section{Participants}

Nine competitive adolescent backstroke swimmers (5 males and 4 females; age: $15.4 \pm 1.7$ years; $50-\mathrm{m}$ backstroke International Swimming Federation [FINA, Fédération internationale de natation] points: $346 \pm$ 142 ; mean \pm standard deviation) volunteered to participate in this study. All swimmers were regularly training 4-6 sessions per week, covering 3-5 km per session and totalling ca. $6-10$ hours per week. The subjects had all been competing for at least 5 years and currently competed at a regional or national level. All swimmers bar one were undertaking an hour of dryland training once a week. Before the study, the individuals' cardiovascular fitness degraded owing to detraining during a 2-month national lockdown caused by the global pandemic. Therefore, we ensured that they all had resumed training for over 2 months before starting the current study. As such, this intervention was conducted during a general preparatory phase of the season in which swimmers were experiencing a gradual build-up of residue fatigue.

\section{Procedures}

The main objective of this pilot study was to investigate if a combined in-water resisted and assisted training program would enhance 50- and 100-m adolescent backstroke performance. Bungee stretch cords were used for the assisted training and a variable-resistance sprint machine was applied for the resisted training.
As other researchers observed positive training adaptations in as little time as 3 weeks, we chose this length of intervention for the training period $[2,14,15]$.

A familiarization session of both training and testing protocols was held the week before baseline testing. Pre- and post-intervention 50- and 100-m backstroke time trials were undertaken, while post-testing was performed within 48 hours of intervention completion. The combined in-water assisted and resisted training program was conducted 3 times per week for 3 weeks. Each session included three $25-\mathrm{m}$ assisted and three 25-m resisted backstroke sprints. Assisted backstroke sprints were performed with a coach using a maximally extended bungee cord (StrechCordz, Tallmadge, OH, USA) to pull a swimmer in from the end of the $25-\mathrm{m}$ pool lane as fast as possible. Simultaneously, the swimmer was instructed to maximize their backstroke arm turnover speed while also kicking as fast as possible. Resisted backstroke sprints were undertaken with swimmers tethered, via a belt worn around the pelvis, to a variable resistance machine (Run Rocket, San Antonio, TX, USA) that was set at a low resistance of between 2 and 6 (out of 30 possible resistance settings). The participants were instructed to swim as fast as possible away from the machine, while the machine provided a constant resistance throughout each $25-\mathrm{m}$ sprint. Each of the 6 sprints (3 assisted and 3 resisted) was interspersed with a 1-minute rest period. During the testing and training intervention sessions, all subjects firstly undertook their standardized individual warm-up procedure as directed by the coach, and the intervention was then incorporated at the beginning of the swimming session.

\section{Measurement protocols}

Backstroke time trials of 50- and 100-m were undertaken at week 0 and week 4 in an indoor 25-m pool with the water temperature maintained at $27.5^{\circ} \mathrm{C}$. Pre- and post-intervention testing was undertaken at the same time of the day, in the early evening (5-7 p.m.). After a thorough individualized race warm-up of at least 20 minutes, the swimmers undertook a maximal 100-m backstroke effort, then had a 10-minute rest interval before completing a 50-m maximal backstroke effort. This rest interval was chosen to replicate the minimal rest time between races that these swimmers could expect to encounter in local swim meets. Furthermore, a 10-minute rest period has been deemed more favourable to performance in comparison with 20 minutes, even in freestyle [16]. The swimmers were stopwatch timed (Finis 3X100M stopwatch; Finis, Inc., 
Livermore, CA, USA) by 2 experienced coaches, with the mean time calculated. Timed splits from each $25-\mathrm{m}$ mark were also measured, as was SR, by using the 3-complete-stroke-cycle method [17]. Immediately after each sprint, the swimmers climbed out of the pool and had their heart rate (HR) measured. Specifically, HR was evaluated with a Polar FS1 HR monitor (Polar Electro, Kempele, Finland) and a Polar T31 HR chest belt attached to a handheld swim bar (Polar Electro, Kempele, Finland), which was pushed against the swimmer's chest. The swimmer's rating of perceived exertion (RPE) was also collected at this time by using Borg's (6-20) scale.

\section{Statistical analysis}

Descriptive data were analysed (mean \pm standard deviation) for all variables, while the magnitude and direction of the difference between pre- and post-intervention testing were calculated with the use of a standardized effect size statistic. Hopkins' criteria were employed for interpreting the effect size: $<0.2$ : trivial, 0.2-0.6: small, 0.6-1.2: moderate, 1.2-2.0: large, 2.04.0: very large, and 4.0+: extremely large [18]. Finally, a one-tailed paired $t$-test for dependent samples was performed on the pre- and post-intervention scores for all normally distributed variables. Most analyses were undertaken with the Apple Numbers software (version 10.3.9). GIGAcalculator.com was used to perform normality testing with the Shapiro-Wilk test, and the Wilcoxon signed-rank testing for non-normally distributed samples was conducted with www.socscistatistics.com.

\section{Ethical approval}

The research related to human use has complied with all the relevant national regulations and institutional policies, has followed the ethical standards of the University of Canterbury Human Ethics Com- mittee and the tenets of the Declaration of Helsinki, and has been approved by the University of Canterbury Human Ethics Committee (approval No.: HEC 2018/88).

\section{Informed consent}

Informed consent has been obtained from all individuals included in this study and their legal guardians.

\section{Results}

Over the 3 weeks, the participants swam the $50-\mathrm{m}$ backstroke trivially faster by $0.4 \pm 1.2 \mathrm{~s}(1.0 \pm 3.1 \%$, $E S=0.07, p=0.19$ ) but this was deemed statistically non-significant. There was over twice as much improvement in the second $25-\mathrm{m}$ split as the first $(1.3 \pm 3.8 \%$ vs. $0.5 \pm 4.2 \%$ ), yet both split times were deemed to have had trivial changes (ES: 0.04 and 0.10 ) from pre- to postintervention testing. SR increased by $0.4 \pm 3.0$ strokes per minute in the post-intervention test, but this was a non-significant trivial change $(E S=-0.07, p=0.33)$. The swimmers' immediate post-swim HR trivially and non-significantly dropped by $3 \pm 18$ beats $\cdot \min ^{-1}(0.4 \pm$ $12.2 \%, E S=0.11, p=0.32$ ) The $50-\mathrm{m}$ perceived exertion was deemed to have a non-normal distribution; however, the Wilcoxon signed-rank test indicated that the pre-intervention score was not statistically significantly higher than the post-intervention one, at the $p<0.05$ level of significance $(Z=-0.3381, p=0.822)$.

For the 100-m backstroke, a significant small improvement was observed, with swimmers becoming $2.9 \pm 2.8 \mathrm{~s}$ faster $(3.4 \pm 3.4 \%, E S=0.27, p=0.01)$. There was an almost uniform improvement across all 4 of the 25-m split times (Figure 1), and the subjects presented a small but non-significant $(p=0.06)$ increase in SR by $1.0 \pm 1.8$ strokes per minute $(3.3 \pm 5.8 \%$, $E S=0.34)$. Their immediate post-swim HR exhibited a small but statistically significant drop of $8 \pm 13$ beats $\cdot \min ^{-1}(4.7 \pm 7.3 \%, E S=0.50, p=0.04)$, while

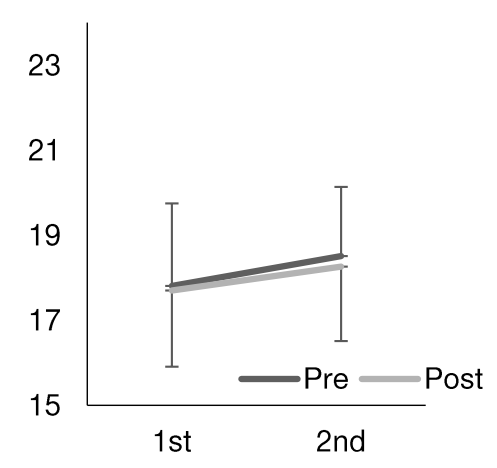

a)

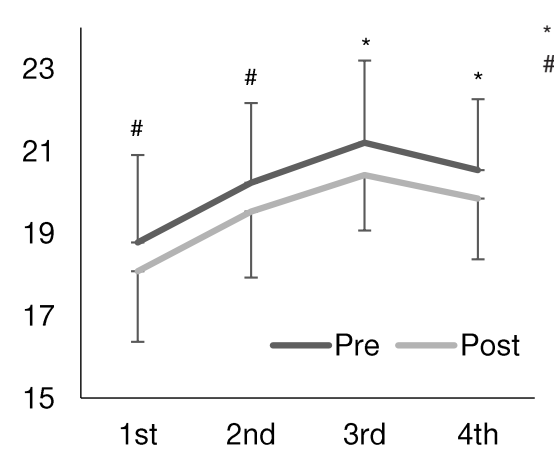

b) $\quad 25 \mathrm{~m}$ split statistically significant change from pre-intervention test at $p<0.05$ \# statistically significant change from pre-intervention test at $p<0.01$

Figure 1. The 25-m split times for (a) 50-m backstroke and (b) 100-m backstroke time trials 
K.A.S. Al-Shdoukhi, C. Petersen, J. Clarke, Resisted and assisted in-water training

Table 1. The 50- and 100-m backstroke time trial data

\begin{tabular}{|c|c|c|c|c|}
\hline Distance & $\begin{array}{l}\text { Before intervention, } \\
\text { week } 0\end{array}$ & $\begin{array}{c}\text { After intervention, } \\
\text { week } 3\end{array}$ & $\begin{array}{l}\text { \% change } \\
\text { (pre-post) }\end{array}$ & $\begin{array}{l}\text { Effect size } \\
\text { (pre-post) }\end{array}$ \\
\hline \multicolumn{5}{|l|}{ 50-m backstroke } \\
\hline 50-m time (s) & $36.33 \pm 3.42$ & $35.96 \pm 3.47$ & $1.0 \pm 3.1$ & 0.07 \\
\hline SR $\left(\right.$ strokes $\left.\cdot \min ^{-1}\right)$ & $40.6 \pm 4.6$ & $41.0 \pm 3.2$ & $1.6 \pm 7.4$ & -0.07 \\
\hline RPE (a.u.) & $14.3 \pm 1.8$ & $14.1 \pm 2.0$ & $-0.6 \pm 16.0$ & 0.08 \\
\hline HR $\left(\right.$ beats $\cdot \min ^{-1}$ ) & $166 \pm 22$ & $163 \pm 10$ & $-0.4 \pm 12.2$ & 0.11 \\
\hline \multicolumn{5}{|l|}{ 100-m backstroke } \\
\hline 100-m time (s) & $80.75 \pm 7.55$ & $77.88 \pm 6.20$ & $3.4 \pm 3.4 \#$ & 0.27 \\
\hline $\mathrm{SR}\left(\right.$ strokes $\left.\cdot \min ^{-1}\right)$ & $32.9 \pm 2.0$ & $33.9 \pm 2.1$ & $3.3 \pm 5.8$ & 0.34 \\
\hline RPE (a.u.) & $15.2 \pm 1.92$ & $15.2 \pm 2.05$ & $1.3 \pm 17.2$ & 0.00 \\
\hline HR (beats $\left.\cdot \min ^{-1}\right)$ & $171 \pm 10$ & $163 \pm 14$ & $-4.7 \pm 7.3^{*}$ & 0.50 \\
\hline
\end{tabular}

SR - stroke rate, RPE - rating of perceived exertion, a.u. - arbitrary unit, HR - heart rate

* statistically significant change from pre-intervention test at $p<0.05$

\# statistically significant change from pre-intervention test at $p<0.01$

the perceived exertion did not change (RPE change: $0 \pm 2.4$ a.u., $E S=0, p=0.50)$ (Table 1).

Subgroup analysis revealed that females had a small, $2.4 \pm 2.7 \%(E S=0.27)$ improvement in the $50-\mathrm{m}$ backstroke compared with males, who swam trivially slower by $-0.2 \pm 3.2 \%(E S=-0.01)$. In the $100-\mathrm{m}$ backstroke, females swam moderately faster by $5.0 \pm 2.5 \%$ $(E S=0.80)$, which compared favourably with the trivial $2.0 \pm 3.5 \%$ faster swimming time of males $(E S=0.18)$. Females obtained a trivial $(2.0 \pm 2.9$ strokes per minute, $E S=0.06)$ and moderate $(1.8 \pm 2.5$ strokes per minute, $E S=0.82$ ) increase in SR for the 50- and 100-m backstroke events, respectively, whereas males had only trivial changes in SR for both events.

\section{Discussion}

The purpose of the present study was to quantify the effect of a combined in-water resisted and assisted training program on adolescent backstroke sprint swimming performance. Our data show that from preto post-intervention testing, the participants swam $3.4 \%$ faster (small significant improvement) in the $100-\mathrm{m}$ backstroke and 1.0\% faster (trivial and nonsignificant improvement) in the 50-m backstroke. The current data should be viewed in the context of what is an expected improvement for these swimmers and in addition to what would constitute a beneficial level of improvement.

The annual improvement in adolescent backstroke swimmers has recently been quantified by age and gender for short-course events of 50- and 100-m distances. The mean annual improvement for swimmers aged $15-16$ years is ca. $1-1.2 \%$ for females and ca. 1.9 $2.6 \%$ for males [19]. Furthermore, Stewart and Hopkins [20] have stated that variables changing performance time by as little as $0.5 \%$ will affect a top junior swimmer's placement. With this in mind, our current inwater resisted and assisted training contributed towards an improvement of $1.0-3.4 \%$ in only 3 weeks. Our results are also within the range of the $2 \%$ gain from 3 weeks of training reported by Girold et al. [3] for an in-water resisted trained group of freestyle swimmers (tested in 100-m freestyle). Our study therefore potentially shows the ability of combined resisted and assisted in-water training to substantially fasttrack backstroke swimming improvement.

Addressing the concern that the lack of a control group is a major limitation of the current study, one could assume that we are unable to differentiate between the improvements from normal swim training vs. those from the additive effect of the combined assisted and resisted training. Opportunely, we do know the mean annual improvement for the national population and can make comparisons with the recently published normative data from our laboratory. A reduction in post-exercising HR is a sign that swimmers improved their cardiovascular fitness. The participants did not feel any difference psychologically (based on RPE measures), so we can imply that a similar level of effort was put in during all time trials with a reduced post-exercise HR.

The trivial improvement in the 50-m backstroke performance contrasts with the findings obtained by Girold et al. [3] in their 2007 study, in which a greater $(2.3 \pm 1.3 \%)$ improvement was observed in $50-\mathrm{m}$ 
freestyle performance in swimmers of a regional to national level. With similar methods, albeit in athletics, it has been established that those who undertook high resistance training gained muscular strength but had no improvement in 100-m running performance, whereas high-speed training improved 100-m sprint performance but did not improve muscular strength $[21,22]$. Given that these researchers measured the effect of their training programs on technical parameters, Maglischo et al. [23] hypothesized that these training methods would cause swimming efficiency to be increased. The hypothesis of Girold et al. [3] was that resisted and assisted training methods should cause adaptations contributing to a greater short-term increase in swimmers' sprint velocity.

Strength training may have an effect on swimming biomechanical parameters as propulsive forces in the horizontal direction are maintained with a longer SL, which improves swimming efficiency and is related to swimming velocity. It is well recognized that a reduction of SL during performance is associated with a decrease of swimming velocity. Chollet et al. [24] reported that when the race distance decreased from $200-\mathrm{m}$ to $50-\mathrm{m}$, there was an increase in both the velocity and SR, while SL decreased. Increasing SR relatively more than the corresponding decrease in SL is the only solution for swimmers to improve velocity in sprint events.

In explaining the relatively greater improvement in the 100-m backstroke, we propose that this may be closely connected to the resisted in-water training, requiring the recruitment of more muscle fibres. Subsequently, with the greater muscle activation, the swimmers could theoretically apply a greater propulsive force, which led to an improved SL [25]. Another possibility for the relatively greater improvement in the 100 -m backstroke could be associated with the greater increase in SR $(3.3 \%, p=0.06, E S=0.34)$ compared with the trivially increased SR in the 50-m backstroke $(1.6 \%, p=0.33, E S=-0.07)$. The raised neural activation developed through the assisted training was previously shown to result in an increased SR [26]. In the present study, the increase in SR would likely be caused by the assisted training [23].

The percentage change in the first $25-\mathrm{m}$ split time for the 2 distances (50 and $100 \mathrm{~m}$ ) equalled 3\%. The $100-\mathrm{m}$ time trial was $3.5 \%$ faster at the $25-\mathrm{m}$ split in the post-intervention test compared with the respective improvement of $0.5 \%$ at this mark during the $50-\mathrm{m}$ time trial distance. While possibly the significant improvement in the first 25-m split of $100 \mathrm{~m}$ could be caused by the assisted training, which is assumed to be an effective way of developing higher SR [27], this does not explain why there was not an equal improvement at the 25-m split during the 50-m time trial. An alternative explanation would be that, as the 100-m time trial was undertaken first, maybe the swimmers were not as psychologically prepared for another big effort only 10 minutes after completing the first time trial. However, participation in successive events with limited recovery time is common in local age-group competition meets, which these swimmers were accustomed to [28].

One of the major limitations of the current investigation was that no tracking of sleep or standardization of diet was undertaken. Both sleep and diet can affect a swimmer's performance and these should be included in future investigations to help better understand factors contributing to performance changes.

From pre- to post-intervention testing, the performance times at the 25-, 50-, 75-, and 100-m split distances of the 100-m backstroke performance were faster and improved by $3.5 \%, 3.3 \%, 3.4 \%$, and $3.2 \%$, respectively. Given the consistent improvement across the entire 100-m time trial distance, we propose that the improvement obtained at these split distances is due to the resisted training. Resisted training is reported to enhance sprinting speed [29] and provide an overload stimulus for strength and power output [30] if the maximum explosive force is exerted in the acceleration phase [1].

In the present study, females had a significantly greater, $5.1 \%$ improvement in $100-\mathrm{m}$ backstroke compared with the $2.0 \%$ improvement that males experienced; we therefore believe that there was a trend for the combined in-water resisted and assisted training program to be more effective for females. This finding is in agreement with a previous study by Girold et al. [2] and may indicate differences in performance levels at the start of the training program. Since at the preintervention testing, males were significantly faster than females, it could be more difficult for them to dramatically increase their velocity. A related possible explanation is that females experienced a relatively greater level of sport-specific overload during the training and subsequently benefited more.

Finally, the training was conducted during a general preparatory phase of the season in which swimmers were experiencing a gradual build-up of residue fatigue. It would be sagacious to repeat the current training intervention within a tapering phase, which we predict would maximize the observed gains. Ideally, a control group would provide a more robust indication of differences between combined assisted/resisted training 
and swim-only training within this population. Future studies should also look at utilizing newer assisted and resisted mechanical technology being employed in elite swim programs, such as the 1080 Sprint machine, which provides a smoother, more constant level of resistance and assistance.

\section{Conclusions}

These results would seem to suggest that adding 3 weeks of combined in-water resisted and assisted training to swim training during a preparatory phase may have contributed towards a small improvement in 100-m backstroke performance. The magnitude of this potential change is likely to be essentially beneficial for 100-m backstroke swimmers. Interestingly, the findings from this study suggest that there may be a trend for females to benefit more than males from this type of training, and this should be investigated in future studies. In conclusion, combined in-water resisted and assisted training should be explored further as a potentially beneficial component for inclusion in adolescent backstroke swimming training programs.

\section{Acknowledgements}

The authors would like to thank Coaches Sam Petty, Martin Harris, Matthew Hurley, and Huia Mikara for their technical expertise.

\section{Disclosure statement}

No author has any financial interest or received any financial benefit from this research.

\section{Conflict of interest}

The authors state no conflict of interest.

\section{References}

1. Wibowo R, Sidik DZ, Hendrayana Y. The impact of assisted sprinting training (AS) and resisted sprinting training (RS) in repetition method on improving sprint acceleration capabilities. J Pendidik Jasmani Olahraga. 2017;9(1):79-86; doi: 10.17509/JPJO.v2i1.7971.

2. Girold S, Calmels P, Maurin D, Milhau N, Chatard J-C. Assisted and resisted sprint training in swimming. $\mathrm{J}$ Strength Cond Res. 2006;20(3):547-554; doi: 10.1519/ R-16754.1.

3. Girold S, Maurin D, Dugué B, Chatard J-C, Millet G. Effects of dry-land vs. resisted- and assisted-sprint exercises on swimming sprint performances. J Strength CondRes. 2007;21(2):599-605; doi:10.1519/R-19695.1.

4. Kuffel NE. The relationship of sprint performance to kinetic and kinematic variables during resisted swimming. Master's thesis. La Crosse: University of Wisconsin-La Crosse; 2011.
5. Muniz-Pardos B, Gomez-Bruton A, Matute-Llorente A, Gonzalez-Aguero A, Gomez-Cabello A, Gonzalo-Skok O, et al. Swim-specific resistance training: a systematic review. J Strength Cond Res. 2019;33(10):2875-2881; doi: 10.1519/JSC.0000000000003256.

6. González Ravé JM, Legaz-Arrese A, González-Mohíno F, Yustres I, Barragán R, de Asís Fernández F, et al. The effects of two different resisted swim training load protocols on swimming strength and performance. J Hum Kinet. 2018;64(1):195-204; doi: 10.1515/hukin-20170194.

7. Papoti M, da Silva ASR, Kalva-Filho CA, Gomes Araujo G, Santiago V, Barreto Martins LE, et al. Tethered swimming for the evaluation and prescription of resistance training in young swimmers. Int J Sports Med. 2017;38(2):125-133; doi: 10.1055/s-0042-105017.

8. Kojima K, Brammer CL, Sossong TD, Abe T, Stager JM. In-water resisted swim training for age-group swimmers: an evaluation of training effects. Pediatr Exerc Sci. 2018;30(1):124-131; doi: 10.1123/pes.2016-0230.

9. Gonjo T, Fernandes RJ, Vilas-Boas JP, Sanders R. Upper body kinematic differences between maximum front crawl and backstroke swimming. J Biomech. 2020;98: 109452; doi: 10.1016/j.jbiomech.2019.109452.

10. Malina RM, Bouchard C, Bar-Or O. Growth, maturation, and physical activity. Champaign: Human Kinetics; 2004.

11. Tolfrey K. Maximal intensity exercise and strength training. In: Armstrong N, van Mechelen W (eds.), Paediatric exercise science and medicine. Oxford: Oxford University Press; 2008; 531-550.

12. Behringer M, Vom Heede A, Yue Z, Mester J. Effects of resistance training in children and adolescents: a metaanalysis. Pediatrics. 2010;126(5):e1199-e1210; doi: 10.1542/peds.2010-0445.

13. Harries SK, Lubans DR, Callister R. Resistance training to improve power and sports performance in adolescent athletes: a systematic review and meta-analysis. J Sci Med Sport. 2012;15(6):532-540; doi: 10.1016/j. jsams.2012.02.005.

14. Strass D. Effects of maximal strength training on sprint performance of competitive swimmers. In: Ungerechts BE, Wilke K, Reischle K (eds.), Swimming science V. Champaign: Human Kinetics; 1988; 149-156.

15. Pichon F, Chatard J-C, Martin A, Cometti G. Electrical stimulation and swimming performance. Med Sci Sports Exerc. 1995;27(12):1671-1676.

16. Neiva HP, Marques MC, Barbosa TM, Izquierdo M, Viana JL, Marinho DA. Effects of $10 \mathrm{~min}$ vs. $20 \mathrm{~min}$ passive rest after warm-up on $100 \mathrm{~m}$ freestyle time-trial performance: a randomized crossover study. J Sci Med Sport. 2017;20(1):81-86; doi: 10.1016/j.jsams.2016. 04.012.

17. Lopes TL, Neiva HP, Gonçalves CA, Nunes C, Marinho DA. The effects of dry-land strength training on competitive sprinter swimmers. J Exerc Sci Fit. 2021;19(1): 32-39; doi: 10.1016/j.jesf.2020.06.005. 
18. Hopkins WG, Marshall SW, Batterham AM, Hanin J. Progressive statistics for studies in sports medicine and exercise science. Med Sci Sports Exerc. 2009;41(1):313; doi: 10.1249/MSS.0b013e31818cb278.

19. Alshdokhi K, Petersen C, Clarke J. Improvement and variability of adolescent backstroke swimming performance by age. Front Sports Act Living. 2020;2:46; doi: 10.3389/fspor.2020.00046.

20. Stewart AM, Hopkins WG. Consistency of swimming performance within and between competitions. Med Sci Sports Exerc. 2000;32(5):997-1001; doi: 10.1097/0000 5768-200005000-00018.

21. Delecluse C, Van Coppenolle H, Willems E, Van Leemputte M, Diels R, Goris M. Influence of high-resistance and high-velocity training on sprint performance. Med Sci Sports Exerc. 1995;27(8):1203-1209; doi: 10.1249/00005768-199508000-00015.

22. Delecluse C. Influence of strength training on sprint running performance. Current findings and implications for training. Sports Med. 1997;24(3):147-156; doi: 10.2165/00007256-199724030-00001.

23. Maglischo EW, Maglischo CW, Zier DJ, Santos TR. The effect of sprint-assisted and sprint-resisted swimming on stroke mechanics. J Swim Res. 1985;1(2):27-33.

24. Chollet D, Seifert LM, Carter M. Arm coordination in elite backstroke swimmers. J Sports Sci. 2008;26(7): 675-682; doi: 10.1080/02640410701787791.

25. Behrens MJ, Simonson SR. A comparison of the various methods used to enhance sprint speed. Strength Cond J. 2011;33(2):64-71; doi: 10.1519/SSC.0b013e318210 $174 \mathrm{~d}$.

26. Mero A, Komi PV, Gregor RJ. Biomechanics of sprint running: a review. Sports Med. 1992;13(6):376-392; doi: 10.2165/00007256-199213060-00002.

27. Ebben WP, Kindler AG, Chirdon KA, Jenkins NC, Polichnowski AJ, Ng AV. The effect of high-load vs. high-repetition training on endurance performance. J Strength Cond Res. 2004;18(3):513-517; doi: 10.1519/ R-12722.1.

28. Toubekis AG, Tsolaki A, Smilios I, Douda HT, Kourtesis T, Tokmakidis SP. Swimming performance after passive and active recovery of various durations. Int $\mathrm{J}$ Sports Physiol Perform. 2008;3(3):375-386; doi: 10.1123/ijspp.3.3.375.

29. Sheppard JM, Dingley AA, Janssen I, Spratford W, Chapman DW, Newton RU. The effect of assisted jumping on vertical jump height in high-performance volleyball players. J Sci Med Sport. 2011;14(1):85-89; doi: 10.1016/j.jsams.2010.07.006.

30. Tufano JJ, Amonette WE. Assisted versus resisted training: which is better for increasing jumping and sprinting? Strength Cond J. 2018;40(1):106-110; doi: 10.1519/SSC.0000000000000362. 